IASSNS-HEP-95/43

hep-th/9510180

\title{
Statistical Thermodynamics of a Black Hole in Terms of Surface Fields ${ }^{\dagger}$
}

\author{
Claudio Teitelboim ${ }^{\ddagger}$ \\ Centro de Estudios Cientificos de Santiago, Casilla 16443, Santiago 9, Chile \\ and \\ Institute for Advanced Study, Olden Lane, Princeton, NJ 08540, USA
}

(May 9, 2018)

\begin{abstract}
An action principle for spacetimes with the topology of an Euclidean black-hole is given. The gravitational field is described by the ordinary volume degrees of freedom plus additional surface fields at the horizon. The surface degrees of freedom correspond to diffeomorphisms on the sphere at the horizon and a field of "opening angles". General covariance forces the surface modes to be confined to a box of an unusual exponential shape, whose volume must be specified as part of the definition of the statistical ensemble. This gives rise to the Bekenstein-Hawking entropy.
\end{abstract}

PACS numbers: 0470.Dy, 04.20.Jb, 04.50.Th, 97.60.Lf

\footnotetext{
${ }^{\dagger}$ A preliminary version of this paper has been cited under the title, "Action principle, state counting and statistical thermodynamics of black holes".

‡Electronic address: teitel@cecs.cl
} 
The interpretation of the black-hole entropy of Bekenstein and Hawking, as the logarithm of the number of states in quantum mechanics or as the logarithm of the available phase space volume in classical mechanics, has been an ongoing dilemma in gravitation theory. The purpose of this paper is to propose a solution to this problem, which may be regarded as formally complete at least within the limits of validity of Einstein's theory itself.

The central idea is to link the entropy with the surface degrees of freedom which give rise to "off-shell" conical singularities at the horizon for Euclidean black-hole geometries. This idea was previously considered by Susskind [1], Bañados, Teitelboim and Zanelli [2], and Carlip and Teitelboim [3], but was not developed to completion. What was missing in the previous analysis was: (i) A complete action leading upon path integration to the transition amplitude between an initial and a final state of the gravitational field in the exterior of a black hole, whose trace gives the partition function. (ii) A determination of the entropy in terms of the density of states, both classical and quantum, i.e., a proper definition of the ensemble at hand. These two steps are taken here.

The action is obtained by analyzing the boundary terms at the horizon arising in the variation of the Hamiltonian action for the gravitational field when the spacetime has the topology of a black hole. One then finds it necessary to include extra surface fields, besides the standard "volume" fields appearing in the action.

Put in classical terms, to account for the black-hole entropy in terms of the surface fields, it is necessary to determine their available phase space. It turns out that the requirement of general covariance fixes the shape of the region of horizon phase space available to the black hole. The size of the region is governed by a single dimensionless parameter $\mathcal{N}$, which is related to the range over which a certain horizon canonical coordinate is allowed to run. This "volume" $\mathcal{N}$ is then held fixed as part of the definition of the ensemble, in addition to the mass and angular momentum (microcanonical ensemble) or in addition to the temperature and rotational chemical potential (grand-canonical ensemble).

One does not see $\mathcal{N}$ in the usual calculations because most of them are done in the semiclassical approximation, where $\mathcal{N}$ appears as a pre-factor which is not analyzed, or 
because the limit of infinite $\mathcal{N}$ is implicitly taken. However, it is crucial for the present analysis to keep $\mathcal{N}$ finite, arbitrary and fixed.

Turn now to the action principle. Since we will not be concerned with the interior of the black hole we will regard the Euclidean formulation as the more fundamental one. This has two important advantages, namely: (i) The "one black-hole sector" is economically defined by demanding that the complete spacetimes have the topology $\mathbb{R}^{2} \times S^{D-2}$. (Extremal black holes require special consideration [4], [5]. See the comments immediately following Eq. (26]) below.) (ii) The solutions of the classical equations of motion (the black holes) are regular everywhere and one can construct an action which has these solutions as bonafide extrema.

To describe the gravitational field in a convenient manner we introduce a polar system of coordinates $\rho, \tau$ in $\mathbb{R}^{2}$. For a classical solution ("on-shell") it is convenient to take the origin $\rho=0$ as the horizon and $\tau$ to be the Killing time. By abuse of language, we will call $\rho=0$ the horizon, even away from the extremum ("off-shell"). The coordinates and metric on $S^{D-2}$ will be denoted by $x^{m}$ and $\gamma_{m n}$ respectively.

Near the horizon one may take the metric to be given by

$$
d s^{2}=d \rho^{2}+\rho^{2} \theta^{2} d \tau^{2}+\gamma_{m n}\left(d x^{m}+N^{m} d \tau\right)\left(d x^{n}+N^{n} d \tau\right)
$$

Here $\theta\left(x^{m}, \tau\right)$ is the proper angle (proper length divided by proper radius) of an arc in $\mathbb{R}^{2}$ of very small radius, centered at $\rho=0$ and of coordinate angular opening $d \tau$.

We are interested in keeping the origin fixed, as this is part of the statement of the problem. For this reason we have set in (1) the lapse and the radial shift at the origin, equal to zero

$$
N=N^{\rho}=0, \quad \text { at } \rho=0 .
$$

Note, however, that the projection $N^{m}\left(x^{p}, \tau\right)$ of the shift vector on $S^{D-2}(\rho=0)$ is left arbitrary: we allow for reparametrizations of the sphere at the origin during the course of time. This will be of importance in what follows.

Actually to specify an origin one needs more than (2), which hold for any origin. One 
may fix a particular origin by demanding that at $\rho=0$ the radial momentum $\pi_{\rho}^{\rho}$ and the radial derivative $\gamma^{1 / 2}, \rho$ of the local area in $S^{D-2}$ vanish,

$$
\pi_{\rho}^{\rho}=\gamma^{1 / 2}, \quad \text { at } \quad \rho=0
$$

This is nothing but the statement that the differential of $\gamma^{1 / 2}$ along $\mathbb{R}^{2}$ should vanish,

$$
d_{R^{2}} \gamma^{1 / 2}=0, \quad \text { at } \rho=0
$$

that is $\gamma^{1 / 2}$ should be stationary. For a solution of the equations of motion this happens at the horizon only, which-incidentally-shows that (3) implies (2), at least "on shell".

The Euclidean action for the wedge will be taken to be the sum

$$
I_{\text {wedge }}=I_{\mathrm{vol}}+I_{\text {hor }},
$$

of the canonical action

$$
I_{\mathrm{vol}}=\int_{\rho>0} d \tau d^{D-1} x\left[\pi^{i j} \frac{\partial g_{i j}}{\partial d \tau}-N \mathcal{H}-N^{i} \mathcal{H}_{i}\right]
$$

and a surface action for the horizon that we will be determined presently.

To determine $I_{\text {hor }}$ one analyzes the surface terms at $\rho=0$ in the variation of $I_{\mathrm{vol}}$. The surface term arises from integrations by parts in the variation of $\mathcal{H}$ and $\mathcal{H}_{i}$, and when $\pi_{\rho}^{\rho}$ and $\gamma^{1 / 2}, \rho$ are held fixed, is given by

$$
\int_{\rho=0} d \tau d^{D-1} x\left[-\theta \delta p-N^{m} \delta \pi_{m}\right]
$$

where

$$
p=\left.(8 \pi G)^{1} \gamma^{1 / 2}\right|_{\rho=0}
$$

and

$$
\pi_{m}=-\left.2 \pi_{m}^{\rho}\right|_{\rho=0}=\left.2(\gamma / g)^{1 / 2} \pi_{m}^{i} n_{i}\right|_{\rho=0}
$$

is the normal-tangential projection at the origin of $\pi^{i j}$ on $S^{D-2}$, (the vector $n_{i}$ is the outward normal to $S^{D-2}$ at a given time $\tau$ ). 
Now, although we might keep $\gamma^{1 / 2}$ and $\pi_{m}$ to be fixed at the origin for all $x, \tau$, it is best to analyze the action when their conjugates are left fixed, as this enables geometrical insight to be gained. In order to do this we must improve the action at $\rho=0$ in a manner analogous to the standard improvement of $I_{\mathrm{vol}}$ at large distances which brings in the mass and angular momentum [6].

We thus add to the action a surface term

$$
\int d \tau d^{D-1} x\left(\theta p+N^{m} \pi_{m}\right)
$$

In the improved action one holds fixed $\theta\left(x^{m}, \tau\right)$ and $N^{m}\left(x^{m}, \tau\right)$. Actually, due to the invariance of the action under surface deformations (changes of the spacetime coordinates) the action will only depend on the total spatial diffeomorphism $f^{M}$ induced by the sequence of shifts $N^{m}(x, \tau)$ and on the total arc $\Theta$ measured perpendicularly to the time constant surfaces. The functions $f^{m}$ and $\Theta$ are related to $\theta$ and $N^{m}$ by

$$
f_{M}^{m} \dot{f}^{M}=N^{m}
$$

and

$$
\dot{\Theta}-\Theta_{, m} f_{M}^{m} \dot{f}^{M}=\theta
$$

Here the dot denotes a partial derivative with respect to $\tau$ and $f_{M}^{m}$ is the inverse of the Jacobian matrix $f^{M},{ }_{m}$.

We bring $\Theta$ and $f^{M}$ into the action by writing $\theta$ and $N^{m}$ in terms of them through (11) and (12). This gives the surface action

$$
I_{\text {hor }}=\int d \tau d^{D-2} x\left[p \dot{\Theta}+f_{M}^{m}\left(\pi_{m}-p \Theta,,_{m}\right) \dot{f}^{M}\right]
$$

If we denote by $p_{M}$ the conjugate momentum to $f^{M}$ we see from (12) that

$$
\pi_{m}=p \Theta,_{m}+p_{M} f^{M}{ }_{m},
$$

which we recognize as the generator of spatial reparametrizations of the scalar fields $\Theta, f^{M}$ and their conjugates $p, \pi_{M}$. This was to be expected since (7) exhibits $\pi_{m}$ as conjugate to the surface shift $N^{m}$. 
It is important to realize that $\pi_{m}$ is not constrained to vanish. This means that the reparametrizations of the $S^{D-2}$ at $\rho=0$ come in not as gauge symmetries, but rather as global symmetries ("improper gauge transformations"). Similarly for the action (13) there is no analog of $\mathcal{H}=0$ at $\rho=0$.

The surface fields $p$ and $\pi_{m}$ may be regarded as the boundary data for the constraint equations $\mathcal{H}_{i}=0$ and $\mathcal{H}=0$. Indeed $\mathcal{H}_{i}=0$ considered as a differential equation in the radial coordinate needs the $\pi_{i}^{\rho}$ to be specified at $\rho=0$. Of these, $\pi_{\rho}^{\rho}$ is set equal to zero on account of (3) leaving $\pi_{m}^{\rho}$ open. Similarly, $\mathcal{H}=0$ needs $\gamma^{1 / 2}$ and $\gamma^{1 / 2},{ }_{\rho}$ to be prescribed at the origin. Of these $\gamma^{1 / 2}, \rho$ is set equal to zero by (3) leaving $\gamma^{1 / 2}$ open.

The equations of motion derived by extremizing $I_{\mathrm{vol}}+I_{\text {hor }}$ are Einstein's equations and, in addition,

$$
\begin{array}{r}
\dot{p}-\left(p N^{n}\right)_{{ }_{n}}=0, \\
\dot{\pi}_{m}-\left[\left(\pi_{m} N^{n}\right)_{{ }_{n}}+\pi_{m, n} N^{n}+\pi_{n} N,_{m}^{n}\right]=0,
\end{array}
$$

which express, that up to a spatial reparametrization $p$ and $\pi_{m}$ are conserved. Here $N^{n}$ is given by (11). Note that the role of the equations of motion for $\Theta$ and $N^{m}$ is played by the definitions (11) and (12) which - in the present formulation - have been incorporated as identities into the action. Equations (11,12,15, 16) reveal the pairs $(p, \Theta),\left(\pi_{m}, f^{M}\right)$ as a sort of action angle variables.

It will be useful in what follows to replace $p$ which is a scalar density, by an invariant $\hat{p}$ which is strictly conserved. This is achieved by the canonical transformation

$$
\begin{array}{r}
\Theta(x, \tau)=\hat{\Theta}(f(x, \tau)) \equiv \hat{\Theta} \circ f \\
p(x, \tau)=J_{f}(x, \tau) \hat{p}(f(x, \tau)) \equiv J_{f} \hat{p} \circ f
\end{array}
$$

where $J_{f}=\operatorname{det}\left(f_{m}^{M}\right)$.

Both $\hat{\Theta}$ and $\hat{p}$ are invariant under reparametrizations. In terms of them the horizon action reads,

$$
I_{\text {hor }}=\int d \tau d^{D-2} x\left[\hat{p} \dot{\hat{\Theta}}+\pi_{m} f_{M}^{m} \dot{f}^{M}\right]
$$


and Eq. (12) becomes just

$$
\dot{\hat{\Theta}} \circ f=\theta \text {. }
$$

The transition amplitude stems from path integrating the action obtained by adding (6) and (19), keeping fixed the fields $\Theta, f$ and $g_{i j}$ at $\tau_{1}$ and $\tau_{2}$. As stated above the result will depend on $\hat{\Theta}, f^{M}$ only through the combinations $\hat{\Theta}\left(\tau_{2}\right)-\hat{\Theta}\left(\tau_{1}\right)$ and $f\left(\tau_{2}\right) \circ f^{-1}\left(\tau_{1}\right)$. It will be convenient in what follows to pass to the representation where $\hat{p}(x)$ is diagonal, rather than $\hat{\Theta}$. This corresponds to adding to the horizon action (19) the total derivative $-\frac{\partial}{\partial \tau}(\hat{p} \hat{\Theta})$. The corresponding amplitude will have the form

$$
\delta\left[\hat{p_{2}}, \hat{p_{1}}\right] K\left[\hat{p_{2}} ; f_{2} \circ f_{1}^{-1} ; \mathcal{G}_{2}, \mathcal{G}_{1}\right]
$$

where $\mathcal{G}$ denotes the $D$-1-dimensional geometry of $g_{i j}$ for $\rho>0$.

The amplitude $K$ appearing in (21) is the result of path integrating the volume action $I_{\mathrm{vol}}$ given by (6) supplemented only by the shift term $N^{m} \pi_{m}$, with $\hat{p}(x, \tau)$ taken equal to $\hat{p}\left(x, \tau_{2}\right)$ for all $\tau$. Note that $\pi_{m}$ is integrated over.

The partition function is is obtained by taking the trace of (21). This amounts to setting $\mathcal{G}_{2}=\mathcal{G}_{1}, f_{2}=f_{1}, \hat{p}_{2}=\hat{p}_{1}$ and integrating over their common values. Observe that setting $f_{2}=f_{1}$ may be simply implemented by taking $N^{m}=0$. Thus the amplitude $K\left[\hat{p_{2}} ; 1 ; \mathcal{G}_{2}=\mathcal{G}_{1}\right]$, where 1 stands for the identity $f(x)=x$, is obtained by path integrating the action $I_{\text {vol }}$ for the closed wedge with the local area element of the $S^{D-2}$ at $\rho=0$ held fixed.

In order to preserve the invariance of the theory under diffeomorphisms of the $S^{D-2}$ at the origin, the integral over $f_{2}=f_{1}$ must be done with an invariant measure over the diffeomorphism group. This brings in the (infinite) volume $V\left(\operatorname{diff}_{S^{D-2}}\right)$ as an overall factor, making the density of configurations of the field $\pi_{m}(x)$ proportional to the volume of the diffeomorphism group, much in the same way as the density of momentum configurations for a particle in a box is proportional to the volume of the box.

On the other hand the the formal symbol $\delta[\hat{p}, \hat{p}]$ is the density of states of the field $\hat{p}(x)$ 
and it is necessary to give meaning to it. This may be done as follows, by invoking general covariance.

One wants the partition function - being a trace - to be independent of the choice of basis in the space of states. In the present case, a choice of basis amounts to a choice of the slicing of spacetime. Now, if one changes the origin in $\mathbb{R}^{2}$ one will change the slicing. Thus one wants the trace to be independent of the choice of origin in $\mathbb{R}^{2}$. (Note that this independence from the origin can only be imposed on the trace and not on the amplitude itself, as the latter does depend on the initial and final states). But this amounts to require that the integrand

$$
\delta[\hat{p}, \hat{p}] \exp \left[\frac{1}{\hbar} I_{\text {vol }}(\text { closed wedge })\right]
$$

be generally covariant (off shell), which means that one must have

$$
\delta[\hat{p}, \hat{p}]=\hat{V} \exp \left(\frac{2 \pi}{\hbar}\right) \int \hat{p} d^{D-2} x
$$

where $\hat{V}$ is a constant.

Indeed the sum

$$
2 \pi \int \hat{p} d^{D-2} x+I_{\mathrm{vol}}=\frac{1}{4 G} \int_{p=0} \gamma^{1 / 2} d^{D-2} x+I_{\mathrm{vol}}
$$

differs from the Hilbert action for the disk by a surface integral at infinity [2].

Thus we learn that the number of surface field configurations between $\pi_{m}$ and $\pi_{m}+d \pi_{m}, \hat{p}$ and $\hat{p}+d \hat{p}$ is given by

$$
\mathcal{N} \exp \left[\left(\frac{2 \pi}{\hbar}\right) \int \hat{p} d^{D-2} x\right] \prod_{x, m} d \pi_{m}(x) d \hat{p}(x)
$$

with

$$
\mathcal{N}=V\left(\operatorname{diff}_{S}^{D-2}\right) \cdot \hat{V}
$$

where $\hat{V}$ is the constant appearing in (23).

If matter gauge fields are present additional surface modes appear in a manner analogous to $\pi_{m}, f^{M}$. They are of the form $\pi_{a}, \Lambda^{a}$, where $\pi_{a}$ is the "electric field density" on the horizon 
and $\Lambda^{a}$ are coordinates on the group manifold. The overall $\mathcal{N}$ is then obtained by multiplying the right side of (26) by $V_{G}$, the product of the volume of the internal gauge group with itself over all points of the sphere.

For extremal black holes the $\mathbb{R}^{2}$ part of the metric in (1) is replaced by $e^{2 \rho} d \tau^{2}+d \rho^{2}$ and the origin, at $\rho=-\infty$, is not on the manifold. As a result $\left(\gamma^{1 / 2}, \Theta\right)$ disappears as an independent canonical pair of surface variables $\left(\Theta\right.$ may be thought of as being zero and $\gamma^{1 / 2}$ becomes a function of $\pi_{m}$ and the gauge momenta $\pi_{a}$ ). Therefore the factor $\delta[\hat{p}, \hat{p}]$ is absent from the trace and $\mathcal{N}$ is given just by $V\left(\operatorname{diff}_{S}^{D-2}\right) \cdot V_{G}$.

In order to understand the meaning of (23) and - in particular - that of the constant $\hat{V}$ in (26 ) it helps to go back to the horizon action (19) and calculate the volume element $\omega$ in the phase space of $(\hat{p}, \hat{\Theta}),\left(\pi_{m}, f^{M}\right)$. One finds

$$
\omega=\prod_{x, m, M}\left[d \hat{p}(x) \wedge d \hat{\Theta}(x) \wedge d \pi_{m}(x) \wedge d f^{M}(x)\right] J_{f}^{-1}(x)
$$

Now, it is immediate to see that

$$
D f=\prod_{x, v} d f^{1}(x) \wedge \ldots \wedge d f^{D-2}(x) J_{f}^{-1}(x)
$$

is an invariant measure over the diffeomorphism group (one has $D(f o h)=D f$ for all $h$ ). Thus the factor $V\left(\operatorname{diff}_{S^{D-2}}\right)$ comes in straight away.

There remains to analyze the integral over $\hat{p}$ and $\hat{\Theta}$. The question to be asked is, how can one achieve that the phase space volume between $\hat{p}(x)$ and $\hat{p}(x)+d \hat{p}(x)$ be of the form (23)? The answer is simple: one needs to properly adjust the region of integration for $\hat{\Theta}(x)$.

To see this it is useful to imagine expanding $\hat{\Theta}$ and $\hat{p}$ in terms of a set of orthonormal functions over the sphere ("spherical-harmonics")labeled by a collective index $i$. Denote by $\hat{\Theta}_{0}, \hat{p}_{0}$ the "zero modes", that is, the integrals of $\hat{\Theta}$ and $\hat{p}$ over the sphere. For fixed $\hat{p}_{i}$ $(i \geq 0)$, the region of integration for $\hat{\Theta}_{i}$ is defined as follows: the zero mode is confined to an "exponential box":

$$
0 \leq \hat{\Theta}_{0} \leq \hat{L}_{0} \exp \left(\frac{2 \pi}{\hbar} \hat{p}_{0}\right)
$$


and the higher modes are contained in an ordinary rectangular box

$$
0 \leq \hat{\Theta}_{i} \leq \hat{L}_{i}, \quad i \geq 1
$$

If we calculate the volume in $\hat{\Theta}, \hat{p}$ space between $\hat{p}_{i}$ and $d \hat{p}_{i}$ we find using(29) and (30) a density of the form (23), with

$$
\hat{V}=\prod_{i \geq 0} \hat{L}_{i}
$$

Thus we see that the exponentially enhanced density (23) may be thought of as due to a peculiar sort of "box" for the zero mode of the $\hat{p}, \hat{\Theta}$ degrees of freedom. The shape of the box is mandated by general covariance. Therefore, we might call (23) the density in the "generally covariant ensemble."

Note that although the preceding analysis is purely classical in language, Planck's constant $\hbar$ is built in the definition (29) of the ensemble which, therefore, is inherently quantum mechanical.

One may also obtain the density (23) by counting eigenvalues. To this effect perform first the canonical transformation

$$
P_{0}=\frac{\hbar}{2 \pi} \exp \frac{2 \pi}{\hbar} \hat{p}_{0}, Q_{0}=\hat{\Theta}_{0} \exp \left(-\frac{2 \pi}{\hbar} \hat{p}_{0}\right)
$$

which involves $\hbar$. Then (29) says that the coordinate $Q_{0}$ is confined to an ordinary box of length $\hat{L}_{0}, 0 \leq Q_{0} \leq \hat{L_{0}}$. If one imposes periodic boundary conditions on this box, the eigenvalues $\left(P_{0}\right)_{n}$ of $P_{0}$ are equally spaced: $\left(P_{0}\right)_{n}=\left(2 \pi \hbar / \hat{L}_{0}\right) n$, with $n$ integer, which means that the eigenvalues $\left(\hat{p}_{0}\right)_{n}$ of $\hat{p}_{0}$ are logarithmically spaced,

$$
\left(\hat{p}_{0}\right)_{n}=\frac{\hbar}{2 \pi} \log \frac{4 \pi^{2}}{\hat{L}_{0}} n
$$

yielding for large $\hat{L}_{0}$ a continuous spectral density of the exponential form (23).

Lastly, observe that for a black hole classical solution $\hat{p}$ and $\pi_{m}$ are expressed in terms of $M$ and $J$ and $I_{\mathrm{vol}}$ vanishes, therefore to the lowest orders in $\hbar$ the entropy reads

$$
S[M, J, \mathcal{N}]=\frac{1}{4 G \hbar} A_{+}(J, M)+\log \mathcal{N}
$$


where $A_{+}$is the area of the horizon.

As long as $\mathcal{N}$ is kept fixed, the $\log \mathcal{N}$ contribution to the entropy has no implications for thermodynamics. However, according to (26) one has,

$$
\log \mathcal{N}=\log V\left(\operatorname{diff}_{S^{D-2}}\right)+\log \hat{V}
$$

and it would seem reasonable that - even after a cutoff is introduced to regularize it $V\left(\operatorname{diff}_{S^{D-2}}\right)$ should be taken to be a universal constant of topological origin. The same applies to the additional $\log V_{G}$ appearing in (35) when gauge matter fields are brought in.

It does not seem equally evident though, that $\hat{V}$ should be treated as universally fixed. Indeed one might imagine, for example, that black holes formed in different ways could acquire $\hat{V}^{\prime} s$ differing from each other by a finite amount. If this were the case, a new thermodynamic parameter for black holes would appear. At the moment of this writing this remains just a speculative note.

The author is grateful to M. Bañados, S. Carlip, D. Gross, F. Wilczek, and J. Zanelli for helpful discussions. This work was supported in part by Grant No. 1940203 of FONDECYT (Chile). Institutional support to CECS from a group of Chilean private companies (COPEC, CGE, Empresas CMPC, ENERSIS, MINERA LA ESCONDIDA, IBM and XEROX) is also acknowledged. 


\section{REFERENCES}

[1] L. Susskind, "Some speculations about black hole entropy in string theory", Rutgers Report, RU-93-94, (1993), (unpublished).

[2] M. Bañados, C. Teitelboim and J. Zanelli, Phys. Rev. Lett. 72957 (1994).

[3] S.Carlip and C. Teitelboim, Class. Quantum. Grav. 121669 (1995).

[4] S. W. Hawking, G. T. Horowitz and S. F. Ross Phys. Rev. D 51, 4302 (1995).

[5] C. Teitelboim, Phys. Rev. D 514315 (1995); Erratum to be published.

[6] T. Regge and C. Teitelboim, Ann. Phys. (NY) 88286 (1974). 